Рубрика: клиническая электрофизиология

( Н.Н. ИЛОВ, А.А. НЕЧЕПУРЕНКО, А.М. АБДУЛКАДЫРОВ, Д.Р. ПАСКЕЕВ, Д.Г. ТАРАСОВ, 2014

( ) АННАЛЫ АРИТМОЛОГИИ, 2014

Удк 616.126.4-089.87:615.84

$\sim$

DOI: 10.15275/annaritmol.2014.2.5

ВЫБОР ВИДА АБЛАЦИИ ПРИ ПРОВЕДЕНИИ ПЕРВИЧНОЙ ПРОЦЕДУРЫ РАДИОЧАСТОТНОЙ АБЛАЦИИ КАВОТРИКУСПИДАЛЬНОГО ПЕРЕШЕЙКА. РЕЗУЛЬТАТЫ ОДНОЦЕНТРОВОГО ПРОСПЕКТИВНОГО РАНДОМИЗИРОВАННОГО ИССЛЕДОВАНИЯ

Тип статьи: оригинальная статья

Н.Н. Илов, А.А. Нечепуренко, А.М. Абдулкадыров, Д.Р. Паскеев, Д.Г. Тарасов

ФГБУ «Федеральный центр сердечно-сосудистой хирургии» Минздрава РФ; ул. Покровская роща, 4, г. Астрахань, 414011, Российская Федерация 
Илов Николай Николаевич, канд. мед. наук, кардиолог, e-mail: nikolay.ilov@gmail.com; Нечепуренко Анатолий Анатольевич, канд. мед. наук, сердечно-сосудистый хирург, заведующий отделением; Абдулкадыров Альберт Мурасбикович, кардиолог; Паскеев Дамир Равильевич, кардиолог; Тарасов Дмитрий Георгиевич, канд. мед. наук, главный врач

Цель. Сравнить клиническую эффективность катетеров с открытым орошением и катетеров с большой длиной дистального кончика (8 мм) при проведении первой процедуры радиочастотной аблации кавотрикуспидального перешейка.

Материал и методы. В исследовании приняли участие 85 пациентов (57 мужчин, 28 женшин) в возрасте 17-78 лет, имевшие документированный пароксизм суправентрикулярной тахикардии с ЭКГ-паттерном типичного трепетания предсердий. Критериями исключения считались декомпенсация хронической сердечной недостаточности, отсутствие венозного феморального доступа, повторная процедура радиочастотной аблации кавотрикуспидального перешейка. Пациенты были рандомизированы в две группы. В основу распределения легло использование в рамках процедуры радиочастотной аблации катетеров различного вида: с открытым орошением (1-я группа) и с большой контактной площадью (2-я группа). Процедура проводилась после получения у пациентов письменного согласия под контролем флюороскопии в соответствии с рекомендациями ведущих зарубежных специалистов. Дизайн исследования подразумевал пошаговое изменение параметров аблации и возможность перехода на другой ее вид. Процедура прекращалась в случае достижения лимита продолжительности операции, принятого равным 3 ч. Учитывались достижение двунаправленного истмус-блока в течение 30 мин аблации (первичная конечная точка) и после 30 мин аблации, время аблации, продолжительность процедуры, время флюороскопии, осложнения, связанные с проведением процедуры (вторичные конечные точки). Для обработки полученного материала и проведения статистического анализа в исследованных группах использовался пакет программ «Statistica» 7,0 (Statsoft).

Результаты. Двунаправленный истмус-блок во время первой процедуры радиочастотной аблации кавотрикуспидального перешейка был верифицирован у 87\% больных. Первичная конечная точка была достигнута у 55 (65\%) пациентов: у 73\% больных в 1-й группе и у 58\% - во 2-й (p=0,002). Была выявлена низкая частота интраоперационных осложнений, сопоставимая в обеих группах.

Заключение. Применение орошаемых катетеров для первой процедуры радиочастотной аблации кавотрикуспидального перешейка позволило получить критерии эффективности процедуры у достоверно большего числа больных. В исследованных группах не было выявлено достоверной разницы в продолжительности процедуры, времени флюороскопии или частоте осложнений, связанных с операцией.

Ключевые слова: кавотрикуспидальный перешеек; орошаемый катетер; катетер с большой контактной площадью.

\title{
TYPE OF PROCEDURE SELECTION IN PRIMARY RADIOFREQUENCY ABLATION OF CAVA-TRICUSPID ISTHMUS. THE RESULTS OF A SINGLE-CENTER PROSPECTIVE RANDOMIZED STUDY
}

\author{
N.N. Ilov, A.A. Nechepurenko, A.M. Abdulkadyrov, D.R. Paskeev, D.G. Tarasov
}

Federal Center for Cardiovascular Surgery of Ministry of Health of the Russian Federation; ulitsa Pokrovskaya Roshcha, 4, Astrakhan', 414011, Russian Federation

Ilov Nikolay Nikolaevich, MD, PhD, Cardiologist, e-mail: nikolay.ilov@gmail.com;

Nechepurenko Anatoliy Anatol'evich, MD, PhD, Cardiovascular Surgeon, Chief of Department;

Abdulkadyrov Al'bert Murasbikovich, Cardiologist;

Paskeev Damir Ravil'evich, Cardiologist;

Tarasov Dmitriy Georgievich, MD, PhD, Head Physician

Objective. To compare the clinical efficacy of the catheters with open irrigation and the large-tip catheters $(8 \mathrm{~mm})$ during the first procedure of radiofrequency ablation of the cava-tricuspid isthmus.

Material and methods. The study involved 85 patients (57 men, 28 women) aged from 17 to 78 years who had a documented paroxysm of supraventricular tachycardia with ECG pattern of a typical atrial flutter. Exclusion criteria considered decompensated heart failure, lack of femoral venous access, a second procedure of radiofrequency ablation of the cava-tricuspid isthmus. Patients were randomized into two groups. The basis of allocation was formed by the implementation in the procedure of radiofrequency ablation catheters of various types: open irrigation cathetrs (first group) and catheters with a large contact area (second group). The procedure was carried out after obtaining of a written consent from the patients, under fluoroscopic control in accordance with the recommendations of leading international experts. The study design meant a step changing of the ablation parameters and switching to the other kinds of them. The procedure was stopped after 
achieving the limit of the duration of the operation, which was equal to three hours. Achievement of bidirectional isthmus block during 30 minutes of the ablation (primary endpoint) and 30 minutes after the ablation, ablation time, duration of the procedure, fluoroscopy time, complications, associated with the procedure, (secondary endpoints) were taken into account. For the processing of the resulting material and statistical analysis in study groups the "Statistics" 7,0 (Statsoft) software package was used.

Results. Bidirectional isthmus block during the first procedure of radiofrequency ablation of the cava-tricuspid isthmus was verified in $87 \%$ of patients. The primary endpoint was achieved in 55 patients (65\%): $73 \%$ of the patients in the first group and $58 \%$ - in the second $(p=0.002)$. A low frequency of intraoperative complications, comparable in both groups was found.

Conclusion. The use of the irrigated catheters for the first procedure of radiofrequency ablation of cava-tricuspid isthmus allowed to obtain criteria of the procedures efficiency on a large number of patients. In the studied groups there were no significant differences in the duration of the procedure, fluoroscopy time or the frequency of the complications associated with the surgery.

Key words: cava-tricuspid isthmus; irrigated catheter; large-tip catheter.

\section{Введение}

$\mathrm{T}$ ипичное трепетание предсердий (ТП) достаточно распространенный вид аритмии, патогенез которой сводится к классическому представлению о механизме макрориентри. Областью критического замедления проведения возбуждения при типичном ТП является кавотрикуспидальный перешеек (КТП). Конечной целью процедуры радиочастотной аблации (РЧА) считается достижение двунаправленной блокады проведения в указанной анатомической области, верифицированной электрофизиологически [1]. Согласно данным ведущих электрофизиологических лабораторий, процент создания истмус-блока (ИБ) после первой РЧА колеблется от 89 до 95, достигая 100 при проведении повторных процедур.

С момента появления новых видов РЧА представители разных центров стали предпринимать попытки выявить преимущества и возможные недостатки использования разных аблационных катетеров, а в качестве экспериментальной модели традиционно служила РЧА КТП. Полученные независимо друг от друга данные однозначно указывают на превосходство орошаемых катетеров, особенно катетеров с открытым орошением (KOO), а также катетеров с большой длиной дистального кончика (КБК) над традиционными 4-миллиметровыми аблационными электродами [2]. Доказанными преимушествами орошаемой РЧА и РЧА с использованием КБК являются обширная площадь и глубина повреждения тканей [3, 4]. Между тем при их использовании необходим оптимальный контакт электрода с тканью для постоянной подачи необходимой энергии. Катетеры с открытым орошением позволяют подавать энергию более постоянно и устойчиво, охлаждение кончика электрода физиологическим раствором предотвращает его перегревание, сокращая риск формирования тромба и/или обугливания и создавая более глубокие, обширные повреждения [5].

Имеющиеся данные о сравнении разных видов РЧА при проведении РЧА КТП бывают противоречивы. Проведенные исследования тяжело сопоставлять ввиду различия в протоколах и неоднозначного методологического подхода при проведении процедуры. Принимая во внимание высокую частоту ТП, имеющуюся разницу в стоимости катетеров, необходимость наличия дополнительного оборудования для использования разных катетеров, можно констатировать, что вопрос выбора оптимального катетера является актуальным и по сей день. Данное проспективное рандомизированное исследование ставит своей целью сравнить клиническую эффективность КБК (8 мм) и КОО при проведении первой процедуры РЧА КТП.

\section{Материал и методы}

Исследование было выполнено в соответствии со стандартами надлежащей клинической практики (Good Clinical Practice) и принципами Хельсинкской декларации. До включения в исследование у всех участников было получено письменное информированное согласие.

\section{Характеристика пациентов}

В исследовании приняло участие 85 пациентов (57 мужчин, 28 женщин) в возрасте 17-78 лет, имевшие документированный пароксизм суправентрикулярной тахикардии с ЭКГ-паттерном типичного ТП. Критериями исключения были декомпенсация хронической сердечной недостаточности, отсутствие венозного феморального доступа, повторная процедура РЧА КТП. Перед операцией проводилась эхокардиоскопия. Пациенты были рандомизированы в две группы: 1-я - РЧА с использованием КОО, 
Таблица 1

Клинико-демографическая характеристика пациентов

\begin{tabular}{|c|c|c|c|}
\hline Признак & $\begin{array}{c}1-я \text { группа } \\
(n=40)\end{array}$ & $\begin{array}{c}2-я \text { группа } \\
(n=45)\end{array}$ & $p$ \\
\hline Пол (муж/жен), $n(\%)$ & $26 / 14(65 / 35)$ & $31 / 14(76 / 24)$ & $>0,1$ \\
\hline Средний возраст, лет* & $57(51 ; 62)$ & $58(51 ; 62)$ & $>0,1$ \\
\hline Структурная патология сердца $n(\%)$ & $8(20)$ & $6(13)$ & $>0,1$ \\
\hline Артериальная гипертензия, $n$ (\%) & $23(58)$ & $23(51)$ & $>0,1$ \\
\hline Ишемическая болезнь сердца, $n$ (\%) & $10(25)$ & $10(22)$ & $>0,1$ \\
\hline Фракция выброса по Simpson, \%* & $55(52,5 ; 63)$ & $55(50 ; 60)$ & $>0,1$ \\
\hline ЛП, см* & $4(3,7 ; 4,5)$ & $4(3,6 ; 4,5)$ & $>0,1$ \\
\hline ЛП & $5(4,9 ; 5,8)$ & $5(4,9 ; 5,8)$ & $>0,1$ \\
\hline ЛП шир, $\mathrm{cm}^{*}$ & $4(3,9 ; 4,6)$ & $4(3,7 ; 4,5)$ & $>0,1$ \\
\hline Объем ЛП, мл* & $69(56 ; 85)$ & $74(64,5,85,5)$ & $>0,1$ \\
\hline$\Pi_{\text {дл }}, \mathrm{cm}^{*}$ & $5(4,6 ; 5,6)$ & $5(4,7 ; 5,7)$ & $>0,1$ \\
\hline ПП & $4(3,4 ; 4,1)$ & $4(3,3 ; 4,1)$ & $>0,1$ \\
\hline Пароксизмальное ТП, $n(\%)$ & $22(55)$ & $25(56)$ & $>0,1$ \\
\hline
\end{tabular}

Примечание. ЛП - левое предсердие; ПП - правое предсердие;

* Данные представлены в виде Ме (25\%; 75\%).

2-я - с использованием КБК. Группы оказались сопоставимыми по основным клинико-демографическим показателям (табл. 1). Для рандомизации применялся макрос Microsoft Excel, использующий генератор случайных чисел.

\section{Внутрисердечное электрофизиологическое исследование и техника РЧА}

Радиочастотную аблацию выполняли натощак, после предварительной отмены антиаритмических препаратов (на 3-5-й день, а в случае приема амиодарона - через 1 мес). Через бедренные вены в сердце доставлялись два диагностических электрода, один из которых (4- или 10-полюсный электрод «MultiCath» («Biotronic», Германия) устанавливался в коронарный синус, другой (20-полюсный электрод «IsmusCath» («Biosense Webster», США) или «StableMap» («Medtronic», США) располагался вдоль кольца трикуспидального клапана.

Об эффективности процедуры судили по достижению двунаправленной блокады проведения импульса через КТП, которая верифицировалась при последовательной стимуляции из области нижней боковой стенки правого предсердия и устья коронарного синуса.

Методика проведения РЧА соответствовала рекомендациям ведущих зарубежных специалистов. Процедуру проводили под контролем флюороскопии. Аблационный катетер позиционировался на кольце трикуспидального клапана на 4-6 ч в рентгенологической проекции
LAO-40, после чего наносили линейные радиочастотные воздействия в направлении нижней полой вены. В зависимости от результатов рандомизации применяли неорошаемые 8-миллиметровые катетеры «Blazer II» («Boston Scientific», США) либо орошаемые катетеры «EzSteer» или «Celsius Thermocool» («Biosense Webster», США). Использовался генератор «Stockert EP-shuttle» («Stockert», Германия). Время одной аппликации не превышало 300 с. Радиочастотную аблацию проводили при температурном контроле. При использовании КБК доставка РЧ-энергии происходила при лимите температуры в $60{ }^{\circ} \mathrm{C}$, верхний предел мощности составлял 70 Вт. При использовании КОО доставка РЧ-энергии происходила при лимите температуры в $43^{\circ} \mathrm{C}$, верхний предел мощности составлял 40 Вт, скорость орошения изотоническим раствором была равна 22 мл/мин. Между аппликациями скорость инфузии снижалась до 2 мл/мин для исключения тромбирования внутреннего просвета катетера. Орошение осуществлялось с помощью насоса «Cool Flow» («Biosense Webster», США). Во всех случаях во время РЧ-аппликации проводился мониторинг импеданса на аблационном электроде. В случае повышения сопротивления более чем на 25 Ом поставка РЧ-энергии прекращалась. Антикоагулянты во время процедуры не применялись.

При отсутствии эффекта и сохранении на локальной электрограмме (ЭГМ) по линии аблации предсердной активности допускалось увеличение 
мощности до 90 Вт для 2-й группы либо до 45 Вт с контролем по мощности для 1-й группы. По истечении 30 мин процедуру аблации по решению оператора прекращали, продолжали РЧА с использованием того же катетера либо проводили переход на другой вид РЧА. Время аблации рассчитывали как математическую сумму продолжительности всех проведенных РЧ-воздействий.

Операцию прекрашали в случае достижения лимита продолжительности, принятого равным 3 ч. Ее продолжительность соответствовала времени от пункции бедренных вен до момента удаления венозных катетеров.

Первичная конечная точка: достижение двунаправленного ИБ в течение 30 мин аблации. Вторичные конечные точки: достижение двунаправленного ИБ после 30 мин аблации, время аблации, продолжительность процедуры, время флюороскопии, осложнения, связанные с проведением процедуры.

\section{Послеоперационное наблюдение}

Пациенты были выписаны из стационара на 2-й день после процедуры. Послеоперационное наблюдение проводилось бессрочно. Оно заключалось в амбулаторной регистрации ЭКГ, а при появлении жалоб на сердцебиение было рекомендовано проведение холтеровского мониторирования по месту жительства. В случае верифицированного рецидива ТП пациента приглашали на проведение повторной процедуры. Период наблюдения составил 2-7 мес.

После проведения процедуры антиаритмические препараты отменялись, за исключением тех случаев, когда типичное ТП было ассоциировано с ФП. Назначение антикоагулянтов проводилось в соответствии со шкалами $\mathrm{CHA}_{2} \mathrm{DS}_{2}$ VASc и HAS-BLED.

\section{Статистический анализ}

Для обработки полученного материала и проведения статистического анализа в исследованных группах использовался пакет программ «Statistica» 7,0 (Statsoft). Центральные тенденции и дисперсия количественных признаков описаны в виде медианы (интерквартильный размах 25-й и 75-й процентили). Сравнение в исследуемых группах и анализ связи проводили при помощи непараметрических методов (Колмагорова-Смирнова и Спирмена соответственно). Критический уровень достоверности нулевой статистической гипотезы об отсутствии различий был принят равным 0,05 .

\section{Результаты}

\section{Первичная конечная точка}

Радиочастотную аблацию проводили во время пароксизма ТП у 50 (59\%) больных и на синусовом ритме - у 35 (41\%) пациентов. Различий между группами по этому параметру выявлено не было. К 10-й минуте РЧА процент пациентов с верифицированным ИБ был примерно одинаковым в обеих группах (около $23 \%, p>0,1$ ). Данный параметр активно прирастал до 25-й минуты аблации $(p<0,05)$, после этого временного отрезка эффективность операции в группах фактически не изменялась, достигнув своих максимальных значений (рис. 1).

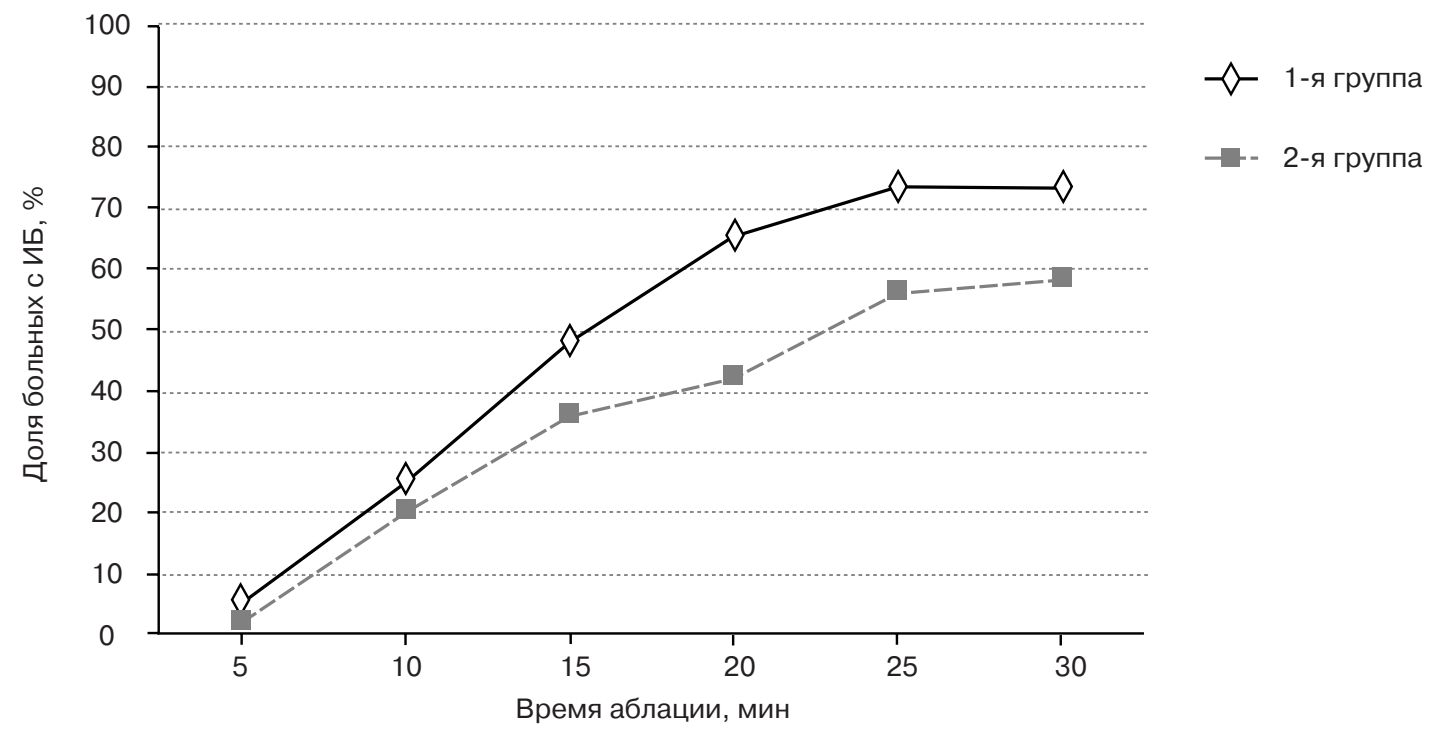

Рис. 1. Взаимосвязь эффективности РЧА и времени аблации 


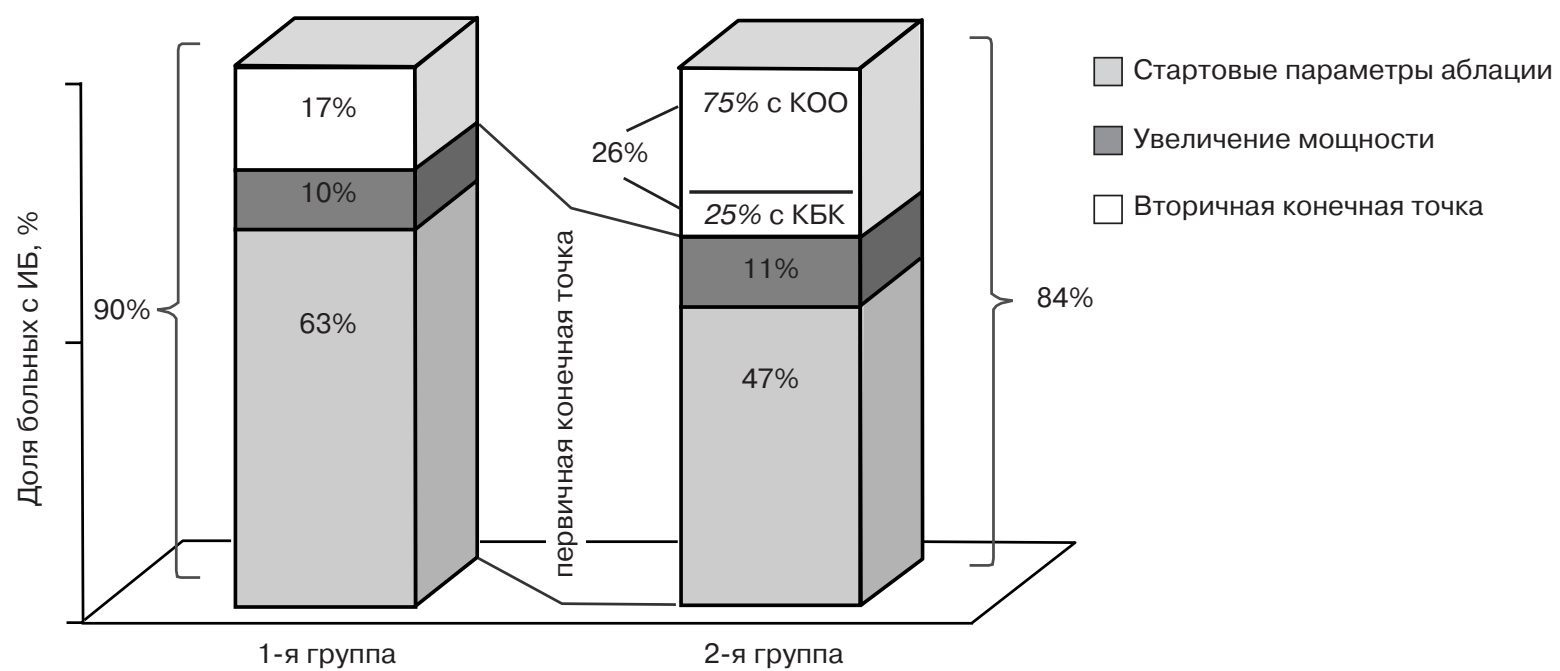

Рис. 2. Процент пациентов с ИБ на разных этапах протокола исследования

Первичная конечная точка была достигнута у 55 (65\%) пациентов: у $73 \%$ больных в 1-й группе и у $58 \%$ - во 2 -й $(p=0,002)$. Для этого в $16 \%$ случаев требовалось перейти на более «жесткий» протокол аблации, прибегнув к контролю по мощности (45 Вт) в 1-й группе (4 пациента) и к увеличению мощности до 90 Вт во 2-й (5 больных).

\section{Вторичные конечные точки}

Согласно протоколу исследования, по истечении 30 мин аблации оператор по своему выбору мог либо продолжить РЧА с применением того же катетера, либо перейти на другой вид РЧА, не превышая установленной общей продолжительности процедуры (3 ч). При использовании исходного катетера критерии ИБ были достигнуты еще у 7 (17\%) больных в 1-й группе и у 3 (6\%) пациентов - во 2-й.
Перехода с орошаемой на традиционную РЧА не проводилось. При изменении вида аблации на орошаемую ИБ удалось верифицировать еще у $20 \%$ больных, доведя суммарный процент успешной аблации во 2-й группе до 84 (рис. 2). Таким образом, по окончании процедур ИБ был верифицирован у 74 пациентов (87\% всех исследуемых больных): у 36 (90\%) больных в 1-й группе и у $38(84 \%)$ - во 2-й.

Время аблации в группах достоверно не различалось: $13,3(7,9 ; 16,5)$ мин в 1-й группе и $13,4(8,9 ; 20)$ мин - во 2-й $(p>0,1)$. При анализе показателей в конце процедуры не было выявлено статистически достоверной разницы ни в общей продолжительности процедуры, ни во времени флюороскопии (табл. 2). Не различались они и по истечении 30 мин РЧА: общая продолжительность операции составила 50 (35; 63)

Особенности радиочастотной аблации в исследуемых группах

\begin{tabular}{l|c|cc}
\hline \multicolumn{1}{c|}{ Признак } & $\begin{array}{c}1-\text { я группа } \\
(n=40)\end{array}$ & $\begin{array}{c}\text { 2-я группа } \\
(n=45)\end{array}$ & $p$ \\
\hline Восстановление СР на воздействии, $n(\%)$ & $11(28)$ & $16(35)$ & $>0,1$ \\
Восстановление СР сверхчастой стимуляцией, $n(\%)$ & $2(5)$ & $4(9)$ & $>0,1$ \\
Восстановление СР после ЭИТ, $n(\%)$ & $8(20)$ & $3(7)$ & $>0,1$ \\
Продолжительность процедуры, мин* & $56(39 ; 82,5)$ & $65(43 ; 85)$ & $>0,1$ \\
Время флюороскропии, мин* & $17(10 ; 24)$ & $16(10 ; 35)$ & $>0,1$ \\
Общая доза, мГр* & $292(136 ; 475,5)$ & $283(160 ; 478)$ & $>0,1$ \\
Кожная доза, мГр* & $3323(1445,5 ; 4811)$ & $3042(1600 ; 5914)$ & $>0,1$ \\
Количество РЧ-воздействий* & $8(5 ; 13)$ & $8(5 ; 19)$ & $>0,1$ \\
Количество РЧ-воздействий до восстановления СР* & $8(4 ; 10)$ & $5(3 ; 8)$ & $>0,1$ \\
\hline
\end{tabular}

Примечание. СР - синусовый ритм; ЭИТ - электроимпульсная терапия.

* Данные представлены в виде Ме (25\%; 75\%). 
и $45(40 ; 63,8)$ мин, время флюороскопии - 14 $(9 ; 19)$ и $12(8 ; 6)$ мин в 1-й и во 2-й группах соответственно (во всех случаях $p>0,1$ ).

В нашем исследовании звуковой феномен «steam рор» был зарегистрирован у 8 пациентов (у 6 больных в 1-й группе и у 2 - во 2-й). У 2 пациентов (по одному в каждой из групп) была диагностирована атриовентрикулярная блокада (АВ-блокада), что потребовало имплантации двухкамерного электрокардиостимулятора. Других осложнений, связанных с проведением процедуры, зарегистрировано не было.

\section{Обсуждение}

\section{Эффективность процедуры}

Возникающие во время аблации острая ишемия и отек ткани могут маскировать жизнеспособный миокард КТП. Через некоторое время после острого повреждения этот участок миокарда может восстановить способность проведения. В $19 \%$ случаев во время процедуры регистрировался транзиторный ИБ, восстановление проведения по правому истмусу наступало через 1,5-10 мин после прекращения РЧА. Рецидив проведения по КТП требовал увеличения мощности (37\%), либо перехода на $\mathrm{KOO} \mathrm{(56 \% ).}$ R. Ventura и соавт. провели похожее по дизайну исследование. При использовании 8-миллиметровых катетеров $\left(65{ }^{\circ} \mathrm{C}, 70\right.$ Вт) и $\mathrm{KOO}\left(65{ }^{\circ} \mathrm{C}\right.$, 50 Вт, 17 мл/мин) у 34\% пациентов интраоперационно регистрировался транзиторный ИБ. Была доказана более высокая эффективность РЧА с КОО именно у данной категории пациентов [6].

В последнее время все чаще появляются публикации, авторы которых подчеркивают важность анатомии КТП при выборе оптимального аблационного катетера [7]. Большинство из них склоняются к мнению, что при «плоском» КТП можно быстрее всего достигнуть ИБ при помощи КБК, в то время как при сложных анатомических вариантах КТП намного предпочтительнее КОО [8]. Предложенные методики визуализации КТП (компьютерная томография, ангиография, 3D-реконструкция, внутрисердечная эхокардиоскопия) ассоциируются с дополнительной лучевой нагрузкой и/или удорожанием стоимости и увеличением времени процедуры [5, 8]. Вероятно, сложность архитектоники КТП может быть определена интраоперационно по невозможности осуществления линейного воздействия. При проведении РЧА с КБК в 22\% случаев мы столкнулись с наличием в области КТП зон, в которых мощность воздействия резко снижалась, а температура в свою очередь повышалась. Такие дискордантные изменения связаны с нанесением РЧ-воздействий в областях со сниженным кровотоком [9], которые не выявляются при «плоском» варианте строения КТП. На наш взгляд, применение конвенционной РЧА в таких случаях оказывается малоперспективным и требует проведения дополнительных аблационных линий в обход таких анатомических регионов либо перехода на орошаемую РЧА.

\section{Безопасность процедуры}

Известно, что при перегреве тканей во время орошаемой аблации возможна регистрация явления «steam pop» [10]. Однако при этом не увеличивается количество осложнений в рамках данной процедуры [11]. В нашем исследовании звуковой феномен «steam рор» был зарегистрирован у 8 пациентов (у 6 больных в 1-й группе и у 2 - во 2-й), в то время как, по данным M. Tokuda и соавт., реальное количество эпизодов такого повреждения можно выявить лишь при использовании внутрисердечного ультразвука [12]. Ни в одном из случаев не было выявлено значимого выпота в перикард и процедура не прекращалась. Нам не удалось обнаружить достоверной связи ни с длительностью процедуры, ни со временем флюороскопии $(p>0,1)$. У одного пациента после регистрации «steam рор» был верифицирован двунаправленный ИБ.

Увеличение площади повреждения тканей во время орошаемой аблации КТП теоретически увеличивает риск повреждения прилегающих к зоне аблации элементов проводящей системы сердца. В эксперименте было показано, что температурный контроль значительно повышает безопасность орошаемой аблации, но достигается это ценой уменьшения площади повреждения, которая значительно выше при фиксированной мощности [13]. Группа авторов из ТельАвива, проанализировав 845 подобных процедур с использованием как орошаемой, так и конвенционной РЧА, описали возникновение АВ-блокады в 1,9\% случаев, связав это с действием радиочастотной энергии либо с механическим воздействием катетеров во время процедуры. При этом лишь 0,6\% пациентов, большая часть из которых имела полную блокаду левой ножки пучка Гиса, потребовалась имплантация постоянного электрокардиостимулятора [14]. В соот- 
ветствии с полученными нами данными АВ-блокада - редкое осложнение РЧА КТП, которое было диагностировано интраоперационно и по результатам последующего холтеровского мониторирования ЭКГ только у 2 пациентов (по одному в каждой из групп). Больному из 1-й группы (1\%) вследствие развившейся постоянной АВ-блокады II ст. (Мобитц II) был имплантирован двухкамерный электрокардиостимулятор. При проведении суточного мониторирования ЭКГ у пациента из 2-й группы была зарегистрирована АВ-блокада I ст., эпизоды транзиторной АВ-блокады II ст. (Мобитц I) преимущественно в ночные часы. По этой причине было принято решение отказаться от имплантации электрокардиостимулятора. Пациенту рекомендовали динамическое наблюдение по месту жительства.

\section{Применение КОО у больных с сердечной недостаточностью}

Проведение орошаемой аблации ассоциируется с введением дополнительного объема физиологического раствора, который, по мнению K.J. Ilg и соавт., составляет около 500 мл за одну процедуру [15]. Согласно нашим данным, для орошения аблационного катетера использовалось в среднем 450 мл физиологического раствора. По причине того, что 20 больных, принявших участие в исследовании, имели сниженную фракцию выброса левого желудочка, излишняя волемическая нагрузка в сочетании с необходимостью длительного горизонтального положения во время операции (продолжительность процедуры составила 60 (41; 85) мин) могла привести к декомпенсации сердечной недостаточности [16]. Обозначенную проблему можно решать либо использованием катетеров с закрытым контуром орошения, либо проведением тщательной медикаментозной подготовки этой категории больных перед процедурой. Определенной альтернативой также может стать использование РЧА с низкой скоростью орошения (10 мл/мин), показавшей высокую эффективность лечения типичного ТП и низкий риск развития осложнений [17].

\section{Предиествовавиие исследования}

Коллектив авторов из Германии в 2002 г. изучил использование 8-миллиметровых катетеров и катетеров с закрытым контуром орошения с параметрами аблации $60-70{ }^{\circ} \mathrm{C}$ (контроль по температуре) и 40-50 Вт соответственно. Про- анализировав 100 процедур РЧА КТП, они не выявили значимой разницы в эффективности этих видов аблации, достигнув двунаправленного ИБ в $99 \%$ случаев [18].

Схожие результаты были достигнуты при сравнении РЧА с 10-миллиметровыми катетерами и КОО [15]. Истмус-блок был верифицирован в $83 \%$ в группе орошаемой РЧА и в $87 \%$ при использовании 10-миллиметровых катетеров. После смены катетеров эти показатели выросли до 90 и 100\% соответственно. Не выявив значимой разницы в клинической эффективности, исследователи подчеркнули меньшую продолжительность процедуры при использовании КБК. На наш взгляд, повлиять на такой вывод мог дизайн исследования, подразумевавший применение орошаемой аблации в сочетании с навигационной системой CARTO.

Практически равная эффективность РЧА с КБК (8 мм) и КОО, составившая 73,8 и 74,2\% соответственно, была показана и другими авторами [19]. В сравниваемых группах проводилась аблация с мощностью 50 Вт, в то время как для достижения главной цели РЧА с КБК нанесения больших по площади участков повреждения - требуется энергия большей мощности [20], что не дает оснований рекомендовать полученные результаты для повседневной практики большинства аритмологических центров.

Группа французских исследователей провела метаанализ, охвативший 603 пациента с ТП. Результаты указывали на равнозначный относительный риск достижения ИБ (отношение шансов 0,96, 95\% доверительный интервал: 0,92-1,01, $p=0,13)$, сопоставимое время РЧА, время флюороскопии и продолжительность процедуры при применении как КБК, так и КОО [21].

Электрофизиологи из г. Бордо (Франция), проводя конвенционную РЧА (8-миллиметровые катетеры с одним и двумя сенсорами, $60{ }^{\circ} \mathrm{C}$, 70 Вт) и орошаемую РЧА (с открытым и закрытым контурами орошения, $50{ }^{\circ} \mathrm{C}, 50 \mathrm{~B}$ ), обнаружили достоверную разницу в конечной точке процедуры. Двунаправленный ИБ был верифицирован в течение 12 мин РЧА у $80 \%$ пациентов, успех операции в группе орошения составил $100 \%$, в группе с 8 -миллиметровыми катетерами $-80 \%$. В отличие от нашего исследования, было подчеркнуто, что использование КОО ассоциировалось с меньшим количеством РЧ-воздействий и коротким временем флюороскопии [22]. 


\section{Ограничения исследования}

Несмотря на то что исследование было рандомизированным, визуальный и тактильный контакт оператора с аблационным катетером не позволял сделать его слепым.

Одним из главных ограничений большинства проведенных ранее похожих исследований было применение РЧА с небольшими параметрами мощности. Использованный нами протокол аблации давал возможность задействовать субмаксимальные параметры РЧА и достигнуть большей степени повреждения морфологического субстрата аритмии.

При отсутствии эффекта через 30 мин аблации не проводился переход на КБК. Это было связано с необходимостью картировать и проводить РЧ-воздействия преимущественно в областях со сниженным кровотоком, что эффективнее осуществлять с помощью 4-миллиметровых КОO.

Анализ стоимости процедуры в исследуемых группах не проводился, поскольку цена на расходные материалы сильно варьирует в разных регионах. Для проведения аблации как с КБК, так и с КОО требуется дополнительное оборудование (генератор, способный вырабатывать высокую мощность, насос для подачи орошающего раствора, расходные материалы и прочее). Как бы то ни было, стоимость процедуры явно возрастает при изменении вида аблации, что стоит принимать во внимание при использовании такой тактики.

\section{Заключение}

Использование КОО и КБК позволяет в подавляющем большинстве случаев достигнуть электрофизиологически верифицированного двунаправленного ИБ во время первой процедуры РЧА КТП (в нашем исследовании - у 87\% больных). Полученные результаты указывают на преимущество орошаемой аблации, позволившей получить критерии эффективности процедуры у большего числа прооперированных больных. На наш взгляд, в основе такого явления лежит возможность КОО доставлять эффективное количество энергии в области со сниженным кровотоком, в которых часто локализуются места «прорыва» электрического возбуждения на КТП. При применении КОО и КБК не было выявлено достоверной разницы в продолжительности процедуры, времени флюороскопии или частоте осложнений, связанных с операцией.

\section{Конфликт интересов}

Во время процедуры использовались диагностические электроды фирм «Biotronic» (Германия), «Biosense Webster» (США) и «Medtronic» (США), аблационные электроды фирм «Boston Scientific» (США) и «Biosense Webster» (США). Исследование было абсолютно независимым и не подразумевало оказание материальной помощи для его проведения ни со стороны фирмпроизводителей, ни со стороны пациентов. Госпитализация и оперативное вмешательство финансировались из федерального бюджета Российской Федерации.

\section{Библиографический список}

1. Takahashi A., Shah D.C., Jaïs P. et al. How to ablate typical atrial flutter. Europace. 1999; 1 (3): 151-5.

2. Tsai C.-F., Tai C.-T., Yu W.-C. et al. Is 8-mm more effective than 4-mm tip electrode catheter for ablation of typical atrial flutter? Circulation. 1999; 100 (7): 768-71.

3. Pérez G.P., Madrid A.H., Rebollo J.M.G. et al. Radiofrequency ablation of the cavotricuspid isthmus in typical atrial flutter: Standard catheter versus irrigated-tip catheter. A Randomized prospective study. Rev. Esp. Cardiol. 2002; 55 (1): 37-44.

4. Cabezaa P., Madrida A.H., Palmeirob A. Lesions produced by radiofrequency ablation of the cavotricuspid isthmus in an experimental model. Rev. Esp. Cardiol. 2003; 56 (10): 963-70.

5. Макарова Н.В., Дурманов С.С., Козлов А.В. и др. Трепетание предсердий: причины неэффективного лечения методом радиочастотной катетерной аблации. Вестник аритмологии. 2012; 69: 57-63.

6. Ventura R., Klemm H., Lutomsky B. et al. Pattern of isthmus conduction recovery using open cooled and solid large-tip catheters for radiofrequency ablation of typical atrial flutter. J. Cardiovasc. Electrophysiol. 2004; 15 (10): 1126-30.

7. Da Costa A., Romeyer-Bouchard C., Jamon Y. et al. Radiofrequency catheter selection based on cavotricuspid angiography compared with a control group with an externally cooled-tip catheter: A randomized pilot study. J. Cardiovasc. Electrophysiol. 2009; 20 (5): 492-8.

8. Koektuerk B., Chun J.K., Wissner E. et al. Cavotricuspid isthmus anatomy determines the success of remote controlled magnetic bidirectional block: A Comparsion between magnetic 8-mm solid tip and 3.5-mm magnetic irrigated tip catheter. Indian Pacing Electrophysiol. J. 2011; 11 (4): 103-14.

9. Hoffmann E., Dorwarth U., Pulter R. et al. Importance of physical parameters for the effectiveness of radiofrequency catheter ablation. Biomed. Tech. (Berl.). 1992; 37 (4): 62-8.

10. Huang S.K.S., Wood M.A. Biophysics of Radiofrequency Lesion Formation. In: Catheter Ablation of Cardiac Arrhythmias. 2nd ed. Elsevier; 2010: 6.

11. Покушалов Е.А. Радиочастотная катетерная аблация трепетания предсердий: автореф. дис. ... д-ра мед. наук. Новосибирск; 2004

12. Tokuda M., Tedrow U.B., Stevenson W.G. Silent steam pop detected by intracardiac echocardiography. Heart Rhythm. 2013; 10 (10): 1558-9.

13. Thiagalingam A., D'Avila A., McPherson C. et al. Impedance and temperature monitoring improve the safety of closed-loop irrigated-tip radiofrequency ablation. J. Cardiovasc. Electrophysiol. 2007; 18 (3): 318-25.

14. Belhassen B., Glick A., Rosso R. et al. Atrioventricular block during radiofrequency catheter ablation of atrial flutter: incidence, mechanism, and clinical implications. Europace. 2011; 13 (7): 1009-14.

15. Ilg K.J., Kühne M., Crawford T. et al. Randomized comparison of cavotricuspid isthmus ablation for atrial flutter using an open irrigation-tip versus a large-tip radiofrequency ablation catheter. J. Cardiovasc. Electrophysiol. 2011; 22 (9): 1007-12. 
16. Akca F., Zima E., Végh E.M. Radiofrequency ablation at low irrigation flow rates using a novel 12-hole gold open-irrigation catheter. Pacing Clin. Electrophysiol. 2013; 36 (11): 1373-81.

17. Новичков С.А. Отдаленные результаты ирригационной радиочастотной катетерной абляции кавотрикуспидального перешейка у больных с типичным трепетанием предсердий: автореф. дис. ... канд. мед. наук. М.; 2005.

18. Schreieck J., Zrenner B., Kumpmann J. et al. Prospective randomized comparison of closed cooled-tip versus 8-mm-tip catheters for radiofrequency ablation of typical atrial flutter. J. Cardiovasc. Electrophysiol. 2002; 13 (10): 980-5.

19. Cuesta A., Mont L., Alvarenga N. et al. Comparison of 8-mmTip and Irrigated-Tip Catheters in the Ablation of IsthmusDependent Atrial Flutter: a Prospective Randomized Trial. Rev. Esp. Cardiol. 2009; 62 (7): 750-6.

20. Feld G. Radiofrequency ablation of atrial flutter using large-tip electrode catheters. J. Cardiovasc. Electrophysiol. 2004; 15 (10 Suppl.): 18-23.

21. Da Costa A. Comparison of the efficacy of cooled-tip and 8mm-tip catheters for radiofrequency catheter ablation of the cavotricuspid isthmus: a meta-analysis. Pacing Clin. Electrophysiol. 2005; 28 (10): 1081-7.

22. Scavée C., Jaïs P., Hsu L.-F. et al. Prospective randomised comparison of irrigated-tip and large-tip catheter ablation of cavotricuspid isthmus-dependent atrial flutter. Eur. Heart J. 2004; 25 (11): 963-9.

\section{References}

1. Takahashi A., Shah D.C., Jaïs P. et al. How to ablate typical atrial flutter. Europace. 1999; 1 (3): 151-5.

2. Tsai C.-F., Tai C.-T., Yu W.-C. et al. Is 8-mm more effective than 4-mm tip electrode catheter for ablation of typical atrial flutter? Circulation. 1999; 100 (7): 768-71.

3. Pérez G.P., Madrid A.H., Rebollo J.M.G. et al. Radiofrequency ablation of the cavotricuspid isthmus in typical atrial flutter: Standard catheter versus irrigated-tip catheter. A Randomized prospective study. Rev. Esp. Cardiol. 2002; 55 (1): 37-44.

4. Cabezaa P., Madrida A.H., Palmeirob A. Lesions produced by radiofrequency ablation of the cavotricuspid isthmus in an experimental model. Rev. Esp. Cardiol. 2003; 56 (10): 963-70.

5. Makarova N.V., Durmanov S.S., Kozlov A.V. et al. Atrial flutter: causes of ineffective radiofrequency catheter ablation. Vestnik Aritmologii. 2012; 69: 57-63 (in Russian).

6. Ventura R., Klemm H., Lutomsky B. et al. Pattern of isthmus conduction recovery using open cooled and solid large-tip catheters for radiofrequency ablation of typical atrial flutter. J. Cardiovasc. Electrophysiol. 2004; 15 (10): 1126-30.

7. Da Costa A., Romeyer-Bouchard C., Jamon Y. et al. Radiofrequency catheter selection based on cavotricuspid angiography compared with a control group with an externally cooled-tip catheter: A randomized pilot study. J. Cardiovasc. Electrophysiol. 2009; 20 (5): 492-8.
8. Koektuerk B., Chun J.K., Wissner E. et al. Cavotricuspid isthmus anatomy determines the success of remote controlled magnetic bidirectional block: A Comparsion between magnetic 8-mm solid tip and 3.5-mm magnetic irrigated tip catheter. Indian Pacing Electrophysiol. J. 2011; 11 (4): 103-14.

9. Hoffmann E., Dorwarth U., Pulter R. et al. Importance of physical parameters for the effectiveness of radiofrequency catheter ablation. Biomed. Tech. (Berl.). 1992; 37 (4): 62-8.

10. Huang S.K.S., Wood M.A. Biophysics of Radiofrequency Lesion Formation. In: Catheter Ablation of Cardiac Arrhythmias. 2nd ed. Elsevier; 2010: 6.

11. Pokushalov E.A. Radiofrequency catheter ablation of atrial flutter. Abstract of the thesis of MD. Novosibirsk; 2004 (in Russian).

12. Tokuda M., Tedrow U.B., Stevenson W.G. Silent steam pop detected by intracardiac echocardiography. Heart Rhythm. 2013; 10 (10): 1558-9.

13. Thiagalingam A., D'Avila A., McPherson C. et al. Impedance and temperature monitoring improve the safety of closed-loop irrigated-tip radiofrequency ablation. J. Cardiovasc. Electrophysiol. 2007; 18 (3): 318-25.

14. Belhassen B., Glick A., Rosso R. et al. Atrioventricular block during radiofrequency catheter ablation of atrial flutter: incidence, mechanism, and clinical implications. Europace. 2011; 13 (7): 1009-14

15. Ilg K.J., Kühne M., Crawford T. et al. Randomized comparison of cavotricuspid isthmus ablation for atrial flutter using an open irrigation-tip versus a large-tip radiofrequency ablation catheter. J. Cardiovasc. Electrophysiol. 2011; 22 (9): 1007-12.

16. Akca F., Zima E., Végh E.M. Radiofrequency ablation at low irrigation flow rates using a novel 12-hole gold open-irrigation catheter. Pacing Clin. Electrophysiol. 2013; 36 (11): 1373-81.

17. Novichkov S.A. Long-term results of irrigated radiofrequency catheter ablation of cavotricuspid isthmus in patients with typical atrial flutter. Abstract of the thesis of PhD. Moscow; 2004 (in Russian).

18. Schreieck J., Zrenner B., Kumpmann J. et al. Prospective randomized comparison of closed cooled-tip versus 8-mm-tip catheters for radiofrequency ablation of typical atrial flutter. J. Cardiovasc. Electrophysiol. 2002; 13 (10): 980-5.

19. Cuesta A., Mont L., Alvarenga N. et al. Comparison of 8-mmTip and Irrigated-Tip Catheters in the Ablation of IsthmusDependent Atrial Flutter: a Prospective Randomized Trial. Rev. Esp. Cardiol. 2009; 62 (7): 750-6.

20. Feld G. Radiofrequency ablation of atrial flutter using large-tip electrode catheters. J. Cardiovasc. Electrophysiol. 2004; 15 (10 Suppl.): 18-23.

21. Da Costa A. Comparison of the efficacy of cooled-tip and 8mm-tip catheters for radiofrequency catheter ablation of the cavotricuspid isthmus: a meta-analysis. Pacing Clin. Electrophysiol. 2005; 28 (10): 1081-7.

22. Scavée C., Jaïs P., Hsu L.-F. et al. Prospective randomised comparison of irrigated-tip and large-tip catheter ablation of cavotricuspid isthmus-dependent atrial flutter. Eur. Heart J. 2004; 25 (11): 963-9.

Поступила 29.09.2014 г. Подписана в печать 23.10.2014 г 